\title{
Virgin Olive Phenolic Profile as a Result of the Anabolic and Catabolic Enzymes Status in the Olive Fruit
}

\author{
Romero-Segura, C., García-Rodríguez, R., Sanz, C., and Pérez, A. G. \\ Department of Physiology and Technology of Plant Products. Instituto de la Grasa \\ (CSIC), Padre García Tejero 4, 41012-Seville, Spain.
}

Keywords: Olea europaea, virgin olive oil, phenolics, $\beta$-glucosidase, peroxidase, polyphenol oxidase

\begin{abstract}
The demand for high quality virgin olive oil (VOO) may be attributed to its potential health benefits related to protection against cancer and cardiovascular diseases and also to its extraordinary organoleptic properties. It is well established that phenolic compounds have a direct influence on both factors, as antioxidants they provide important nutritional benefits, and additionally, some of them are associated with the bitter and pungent sensory notes of the oil. VOO phenolic profile may be affected by multiple preharvest and postharvest factors. In this sense, though processing technology may severely affect VOO quality, it is clear that most quality attributes of the oil are determined by the chemical composition and biochemical status of the olive fruit. Thus, the content of the main hydrophilic phenols found in VOO is closely related to the activity of enzymes hydrolyzing the main phenolic glycosides initially present in the olive tissue and the pool of enzymes, associated to the oxidative catabolism, acting both on those glycosides and on the derived hydrophilic phenols. Management of VOO phenolic profile requires a deeper knowledge on the anabolic and catabolic enzymes features in the olive fruit and the activity pattern of these enzymes during the industrial process to obtain this product.
\end{abstract}

\section{INTRODUCTION}

VOO phenolic composition is determined by the content of phenolic glycosides initially present in the olive tissue and the activity of various hydrolytic enzymes acting on these glycosides as well as endogenous oxidoreductases that promote phenolic oxidation during the crushing and kneading steps in the olive oil extraction process. Among the main phenolic glycosides found in olive fruit are oleuropein, ligstroside and demethyloleuropein, and the aglycon derivatives resulting from their enzymatic hydrolysis, dialdehydic forms of decarboxymethyl oleuropein and ligstroside aglycones (DGO and DGL, respectively), and aldehydic forms of oleuropein and ligstroside aglycones (AGO and AGL, respectively), commonly known as secoiridoid compounds, constitute quantitatively the most significant phenolic components of VOO. The key enzymatic activity controlling the hydrolysis of phenolic glycosides during the olive oil extraction process seems to be an endogenous $\beta$-glucosidase that, according to our data, exhibits maximum activity towards oleuropein (Romero-Segura et al., 2009). On the other hand, a number of previous studies reported that phenolics catabolism, due to chemical and/or enzymatic oxidation, is critical during the kneading step in which the presence of oxygen may reduce by half the concentration of orthodiphenols in oils (García et al., 2001). The main oxidizing agents of phenols in plant products are free radicals and 
oxidoreductase activities such as polyphenol oxidase (PPO) and peroxidase (POX). PPO is the main enzyme involved in the oxidation of phenols, both in physiological processes associated with fruit ripening and in any form of manipulation of the fruit involving tissue damage or breakage. POXs (Class III) oxidize phenolics as preferential substrates at the expense of peroxides, in most cases $\mathrm{H}_{2} \mathrm{O}_{2}$. Although contribution of POX to the oxidation of phenols is limited by the availability of $\mathrm{H}_{2} \mathrm{O}_{2}$, autoxidation of phenolics caused by tissue damage increases $\mathrm{H}_{2} \mathrm{O}_{2}$ content, and this $\mathrm{H}_{2} \mathrm{O}_{2}$ may be used by $\mathrm{POX}$ to enhance oxidation of phenolics. Both enzymes seem to act synergistically. Some previous studies have focused on the roles play by both PPO and POX in olive phenolics metabolism. Thus, it has recently been shown the inverse relationship between levels of PPO and oleuropein content along ripening of the olive fruit (Ortega-Garcia et al., 2008). A central role has also being attributed to this enzyme in the enzymatic browning observed in bruised olive fruits (Segovia-Bravo et al, 2009). On the other hand, high levels of POX activity have been found in olive seed and the effect of this enzymatic activity on the VOO phenolic profile has been proved (Luaces et al., 2007). A POX enzyme that binds specifically to pectic polysaccharides has been purified from black ripened olives (cv. Douro) (Saraiva et al, 2007) and more recently Tzika et al., (2009) have reported the partial purification of a POX enzyme from Koroneiki olive fruits that seems to be active towards some olive fruit phenols but inactive towards oleuropein.

The aim of this study was to investigate whether POX and PPO compete with $\beta$ glucosidase for oleuropein and other phenolic glucosides during the olive oil extraction process, and to study the contribution of PPO and POX to the oxidation of VOO secoiridoid derivatives by directly assaying the activity of these two enzymatic activities with olive natural substrates.

\section{MATERIALS AND METHODS}

\section{Plant material and oil extraction}

Olive fruits (Olea europaea cv. Picual and Arbequina) were cultivated at the experimental fields of Instituto de la Grasa and collected, along two consecutive years, at the appropriated maturity stage. Fruit maturity was expressed in terms of weeks after flowering (WAF).

Extraction of fruit and VOO phenolic compounds to be used as substrates

Fruit phenolics were extracted according to a previously developed procedure (Romero- Segura et al., 2009). Representative samples of fruits $(1 \mathrm{~g})$ were homogenized at $4^{\circ} \mathrm{C}$ with dimethyl sulphoxide $(6 \mathrm{ml})$ by means of an Ultraturrax homogenizer $(2 \times 30 \mathrm{~s}$ at maximum speed). The supernatant obtained after centrifugation at $3000 \mathrm{~g}$ for $2 \mathrm{~min}$, was filtered thorough $0.22 \mu \mathrm{m}$ and used as fruit phenolic extract.

VOO phenolics were isolated by solid phase extraction on a diol-bonded phase cartridge (Supelco, Bellefonte, PA) and analyzed by HPLC following a previously described procedure (Mateos et al., 2001)

\section{Enzyme extraction and purification.}

Acetone powders were prepared from mesocarp and seed tissues of fresh harvested olive fruits. $\beta$-glucosidase was extracted from mesocarp acetone powders and purified according to Romero-Segura et al. (2009).

POX enzyme extracts were prepared from $0.25 \mathrm{~g}$ of seed acetone powder in $2.5 \mathrm{ml}$ of a buffer consisting of $100 \mathrm{mM}$ sodium phosphate buffer, pH 6.7, $1 \mathrm{mM}$ EDTA, 0.1 $\mathrm{mM}$ phenylmethyl sulfonyl fluoride (PMSF), $0.1 \mathrm{mM}$ benzamidine, $5 \mathrm{mM} \alpha$ aminocaproic acid, $0.1 \%$ Triton X-100 (buffer A) using an Ultraturrax homogenizer. The 
resulting homogenate was centrifuged at $10000 \mathrm{~g}$ for 20 min at $4{ }^{\circ} \mathrm{C}$ and the clear supernatant was used as crude extract. These POX extracts are deprived of PPO activity.

PPO enzyme extracts were prepared from $0.25 \mathrm{~g}$ of mesocarp acetone powder in $10 \mathrm{ml}$ of the previously described buffer in which $0.1 \%$ Triton X-100 was substituted by $1 \mathrm{mM}$ sodium dodecyl sulphate (SDS). The homogenate obtained by using an Ultraturrax homogenizer was centrifuged at $27000 \mathrm{~g}$ for $20 \mathrm{~min}$ at $4{ }^{\circ} \mathrm{C}$. To eliminate interferences due to the presence of other enzymatic activities such as $\beta$-glucosidase this crude extract was partially purified by $\left(\mathrm{NH}_{4}\right)_{2} \mathrm{SO}_{4}$ precipitation. The pellet obtained at 35-60\% cut was used as partially purified PPO extract. Neither $\beta$-glucosidase nor POX activity were detected in this partially purified PPO extracts.

\section{Enzyme activity assays}

$\beta$-glucosidase activity was determined by monitoring the increase in absorbance at $405 \mathrm{~nm}$ related to the amount of $\mathrm{p}$-nitrophenol $\left(E=552.8 \mathrm{M}^{-1} \mathrm{~cm}^{-1}\right)$ liberated from the synthetic glucoside p-nitrophenyl- $\beta$,D-glucopiranoside ( $\mathrm{pNPG}$ ). One unit of $\beta$-glucosidase activity was defined as the amount of enzyme able to release $1 \mu \mathrm{mol}$ of $\mathrm{p}$-nitrophenol per $\min$ at $45^{\circ} \mathrm{C}$. The method used to assay $\beta$-glucosidase activity towards olive natural phenolic glycosides was based on the direct determination of the hydrolytic degradation of each glucosidic compound by HPLC analysis (Romero-Segura et al., 2009). Blank samples with thermally denatured enzyme extracts $\left(60 \mathrm{~min}\right.$ at $\left.100^{\circ} \mathrm{C}\right)$ were measured in parallel to quantify and subtract non-enzymatic hydrolysis.

POX activity was spectrophotometrically determined by continuously monitoring the increase in absorbance at $420 \mathrm{~nm}$ related to formation of tetraguaiacol ( $E=26600 \mathrm{M}^{-}$ ${ }^{1} \mathrm{~cm}^{-1}$ ) by peroxidation of guaiacol (Luaces et al., 2007). One unit of POX activity was defined as the amount of enzyme oxidazing $1 \mu \mathrm{mol}$ of guaiacol per min. POX activity towards natural olive phenolics was assessed using fruit phenolic extracts and VOO phenolic extracts as substrates. Remaining phenolics were quantified by HPLC as previously described for $\beta$-glucosidase. The standard reaction medium consisted of 1.5 $\mathrm{mL}$ of $25 \mathrm{mM}$ sodium acetate buffer $\mathrm{pH} 6.5,6.5 \mathrm{mM}$ oleuropein (or VOO phenolics), 2 $\mathrm{mM} \mathrm{H}_{2} \mathrm{O}_{2}$ and the appropriate amount of enzyme $(100-250 \mu \mathrm{L})$. Besides, control samples with thermally denatured enzyme extracts or without $\mathrm{H}_{2} \mathrm{O}_{2}$ were measured in parallel to quantify non-peroxidative degradation of phenolic substrates.

PPO activity was determined by continuously monitoring the increase in absorbance at $400 \mathrm{~nm}$ related to the oxidation of tert-butylcatechol (TBC) and the formation of the corresponding quinone. Quantification of the oxidative reaction was carried out considering an extinction coefficient of $1200 \mathrm{M}^{-1} \mathrm{~cm}^{-1}$ (García-Molina et al., 2007). PPO activity towards olive natural phenolics was assessed using the same methodology previously described for POX. The standard reaction medium consisted of $1.5 \mathrm{~mL}$ of $25 \mathrm{mM}$ sodium acetate buffer, $\mathrm{pH} 6.5,6.5 \mathrm{mM}$ oleuropein (or VOO phenolics), and the appropriate amount of enzyme $(5-50 \mu \mathrm{L})$. Besides, control samples with thermally denatured enzyme extracts or with active enzyme extracts containing a PPO inhibitor, tropolone $(0.2 \mathrm{mM})$, were measured in parallel to quantify any non-oxidative degradation of phenolic substrates.

\section{RESULTS AND DISCUSSION}

As a first approach to evaluate the relative contribution of $\beta$-glucosidase, POX, and PPO to phenolic composition of VOO, the activity profile of these enzymes were analyzed in two olive cultivars, Picual and Arbequina, along fruit ripening (Table 1). 
Olive $\beta$-glucosidase activity increases along fruit ripening and it reaches a maximum at 34 WAF in both cultivars. Then this activity declined abruptly at 38 WAF. However, non significant differences were observed in POX and PPO activity levels in the period 34-38 WAF. Though it is not possible to compare absolute activity values obtained with three different substrates, it is clear that at harvest (34-38 WAF) olive fruits contain a significant pool of the three above mentioned enzyme activities.

Previous experimental findings have demonstrated that olive $\beta$-glucosidase is very active against oleuropein. In fact, kinetic studies carried out with the purified enzyme show that olive $\beta$-glucosidase is 200 -fold more active against oleuropein than against the synthetic substrate pNPG. Using experimental conditions previously described (RomeroSegura et al., 2009), oleuropein hydrolysis by olive $\beta$-glucosidase was monitored along reaction time. After one minute, oleuropein decreases up to $30 \%$ of its initial content and two main hydrolytic products were formed: AGO isomers and AGO (Figure 1). Oleuropein degradation was completed after 3 min of reaction, and AGO was the main compound found at longer incubation times. These data are in good agreement with the phenolic profiles found in olive pastes resulting from the milling of olive fruits. As displayed in Figure 2, immediately after the milling step most of the oleuropein and ligstroside initially present in the olive fruit were metabolized while demethyloleuropein was still detected in olive pastes, and verbascoside, apigenin-7-glucoside and luteolin-7glucoside were metabolized at a lower rate.

To investigate whether POX and PPO contribute to this rapid oleuropein degradation observed during olive oil extraction process a series of incubation experiments were carried out with olive POX and olive PPO using oleuropein and verbascoside as substrates (Table 2). Both oxidoreductases were found to be active against oleuropein. Oleuropein oxidation by POX reached a maximum rate at two minutes, when an average $15 \%$ of the initial oleuropein content was degraded. At longer incubation times the reaction rate slows down and non significant progress is observed after $5 \mathrm{~min}$. The high affinity of olive POX for verbascoside could explain the metabolization of this compound observed in Figure 2. This metabolization could not be attributed to enzymatic hydrolysis by $\beta$-glucosidase since this enzyme is not active against verbascoside according to Romero-Segura et al. (2009).

Partially purified olive PPO also demonstrated to be active towards both substrates, with a slightly higher oxidation rate observed for verbascoside than for oleuropein (Table 2). The highest oleuropein oxidation rates were measured at 2 min of incubation with PPO although degradation of this phenolic glucoside was almost linear throughout 30 minutes of reaction. Experimental data obtained suggest that both, POX and PPO, may contribute to oleuropein degradation during olive processing. Further studies need to be performed to calculate kinetic parameters of oleuropein oxidation with pure POX and PPO enzymes.

After testing the ability of olive POX and PPO to oxidize phenolic glucosides, the activity of these enzymes was assayed using VOO secoiridoid compounds as substrates. DGO and AGO were the preferred substrates for olive POX, that caused a $25 \%$ content decrease of these ortho-diphenolic secoiridoids after 5 minutes of reaction (Figure 3). On the contrary, the concentration of ligstroside derivatives, DGL and AGL, remained unaltered after 10 minutes of incubation (data not shown). As previously observed with oleuropein, non significant progress in the ortho-diphenols oxidation reaction was observed at longer incubation times. On the other hand, the highest PPO oxidation rates were also observed for ortho-secoiridoid compounds, displaying a $40 \%$ decrease in AGO 
and DGO contents after $10 \mathrm{~min}$ of enzyme reaction. Almost no PPO activity was observed towards AGL and DLG. The high specificity of olive PPO for ortho-diphenols confirms that olive PPO mainly exhibits diphenolase activity.

\section{ACKNOWLEDGEMENTS}

We thank M. Pascual for their excellent technical assistance. This work was supported by Research Project AGL2008-00258 from Programa Nacional de Recursos y Tecnologías Alimentarias funded by the Spanish Government.

\section{Literature Cited}

García-Molina F., Muñoz J.L., Varón R., Rodríguez-López J.N. and García-Cánovas F. 2007. A review on sprectophotometric methods for measuring the monophenolase and diphenolase activities of tyrosinase. J. Agric. Food Chem. 55: 9739-9749.

García, A., Brenes, M., Martínez, F., Alba, J., García, P. and Garrido, A. 2001. HPLC evaluation of phenols in virgin olive oil during extraction at laboratory and industrial scale. JAOCS 78:625-629.

Luaces, P., Romero, C., Gutierrez, F., Sanz, C. and Perez, A.G. 2007. Contribution of olive seed to the phenolic profile and related quality parameters of virgin olive oil. J. Sci. Food Agric. 87:2721-2727.

Mateos, R., Espartero, J.L., Trujillo M., Rios J.J., Leon-Camacho, M., Alcudia, F., Cert, A. 2001. Determination of phenols, flavones, and lignans in virgin olive olis by solidphase extraction and high performance liquid chromatography with diode array ultraviolet detection. J. Agric. Food Chem. 49:2185-2192.

Romero-Segura, C., Sanz, C., and Perez, A.G. 2009. Purification and Characterization of an olive fruit beta-glucosidase involved in the biosynthesis of virgin olive oil phenolics. J. Agric. Food Chem. 57:7983-7988.

Saraiva J.A., Nunes C.S. and Coimbra M.A. 2007. Purification and characterization of olive (Olea europea L.) peroxidase. Evidence for the occurrence of a pectin binding peroxidase. Food Chem. 101:1594-1602.

Segovia-Bravo, K.A., Jarén-Galán, M., García-García P. and Garrido-Fernández, A. 2009. Browning reactions in olives: Mechanism and polyphenols involved. Food Chem. 114:1380-1385.

Tzika E.D., Papadimitriou V., Sotiroudis T.G. and Xenakis A. 2008. Oxidation of oleuropein studied by EPR and spectrophotometry. Eur. J. Lipid Sci. Technol. 110:149-157 


\section{$\underline{\text { Tables }}$}

Table 1.- Enzymatic activity level of $\beta$-glucosidase, POX and PPO in olive fruit along ripening

\begin{tabular}{ccccc}
\hline \multirow{2}{*}{ Olive cultivar } & $\begin{array}{c}\beta \text { Ripening stage } \\
(\text { WAF })\end{array}$ & $\begin{array}{c}\beta \text {-Glucosidase } \\
(\mathrm{U} / \mathrm{g} \text { FW })\end{array}$ & $\begin{array}{c}\text { POX } \\
(\mathrm{U} / \mathrm{g} \text { FW })\end{array}$ & $\begin{array}{c}\text { PPO } \\
(\mathrm{U} / \mathrm{g} \text { FW })\end{array}$ \\
\hline Arbequina & 26 & $3.1 \pm 0.8$ & $9.2 \pm 0.8$ & $216.4 \pm 34.3$ \\
& 30 & $4.9 \pm 0.9$ & $17.4 \pm 1.5$ & $191.0 \pm 30.2$ \\
34 & $5.2 \pm 1.3$ & $15.5 \pm 2.2$ & $192.0 \pm 30.4$ \\
38 & $1.8 \pm 0.5$ & $15.0 \pm 1.9$ & $210.4 \pm 33.3$ \\
& 26 & $4.1 \pm 1.1$ & $8.9 \pm 1.1$ & $369.6 \pm 58.5$ \\
30 & $4.9 \pm 1.3$ & $20.5 \pm 2.2$ & $205.2 \pm 32.5$ \\
34 & $10.8 \pm 1.9$ & $20.8 \pm 2.4$ & $204.7 \pm 32.4$ \\
& 38 & $5.1 \pm 1.2$ & $21.8 \pm 2.5$ & $156.9 \pm 24.8$ \\
\hline
\end{tabular}

Table 2.- Relative PPO and POX activity against different substrates

PPO relative activity $\quad$ POX relative activity

\begin{tabular}{lcc} 
Substrate & $(\%)$ & $(\%)$ \\
\hline Guaiacol 5.5mM & - & 100 \\
TBC 7 mM & 100 & - \\
Oleuropein 6.5mM & 31.0 & 29.45 \\
Verbascoside $6.5 \mathrm{mM}$ & 44.5 & 118 \\
\hline
\end{tabular}




\section{$\underline{\text { Figures }}$}
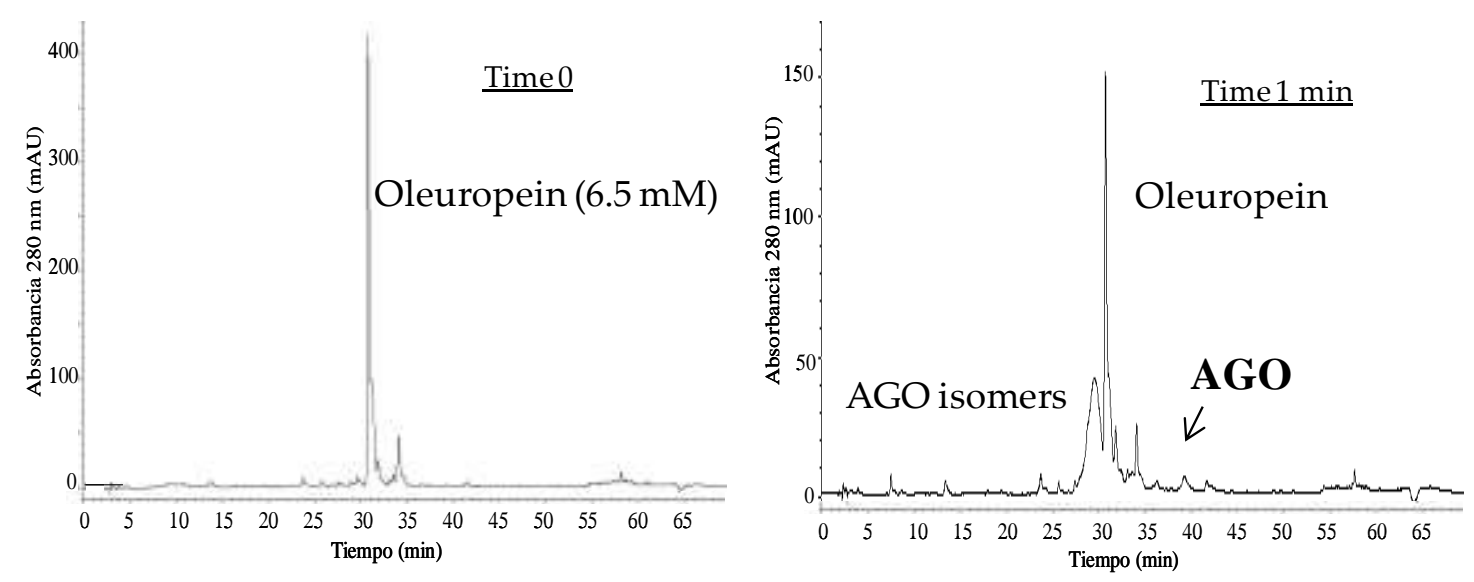

Figure 1.- Oleuropein hydrolysis by olive $\beta$-glucosidase.

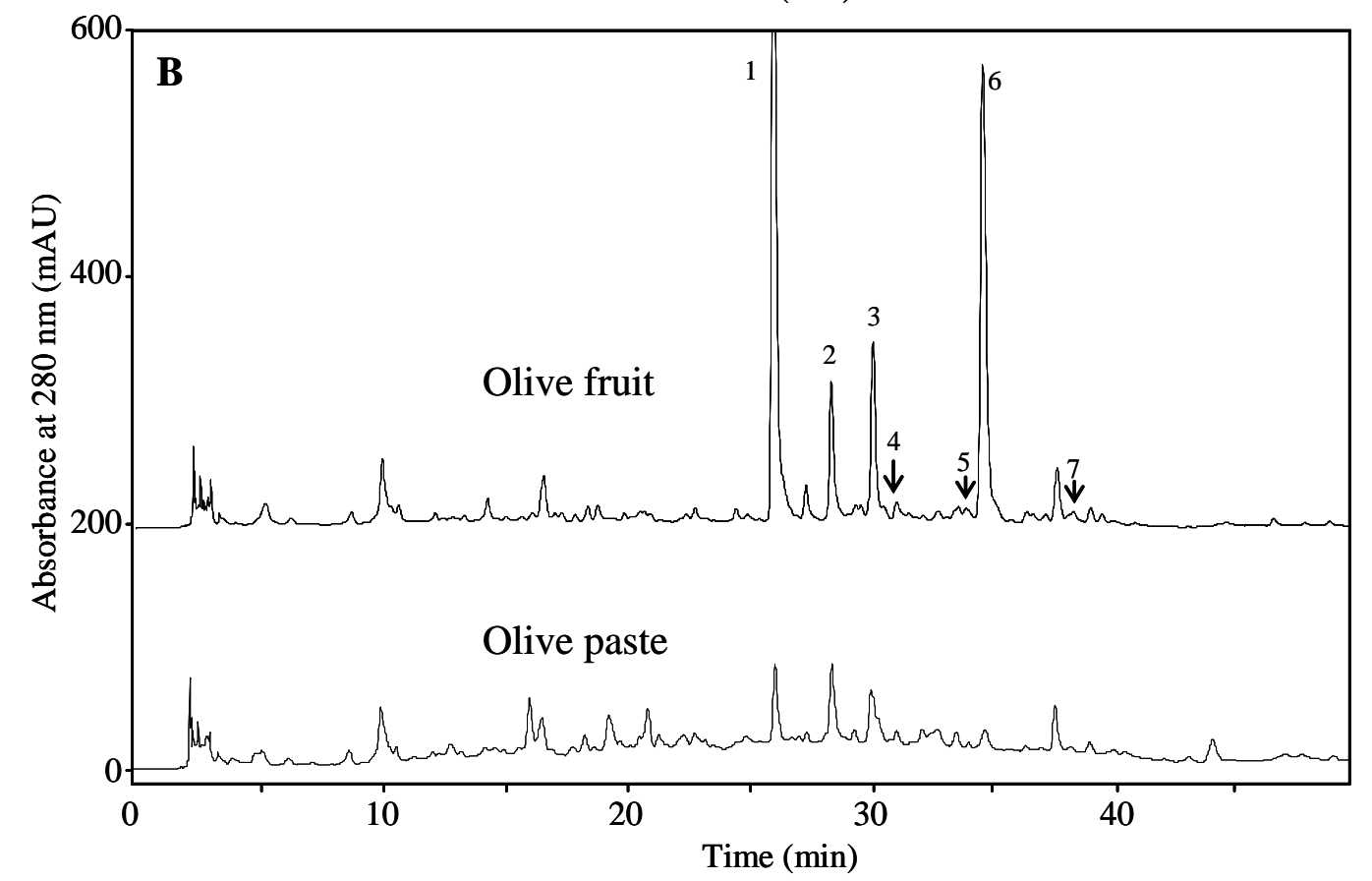

Figure 2.- Phenolic profiles of Arbequina olive fruits and pastes after the milling step in the process to obtain virgin olive oil. Peaks: 1 , demethyloleuropein; 2 , verbascoside; 3 , luteolin-7glucoside; 4, rutin; 5, apigenin-7-glucoside; 6, oleuropein; 7, ligstroside 


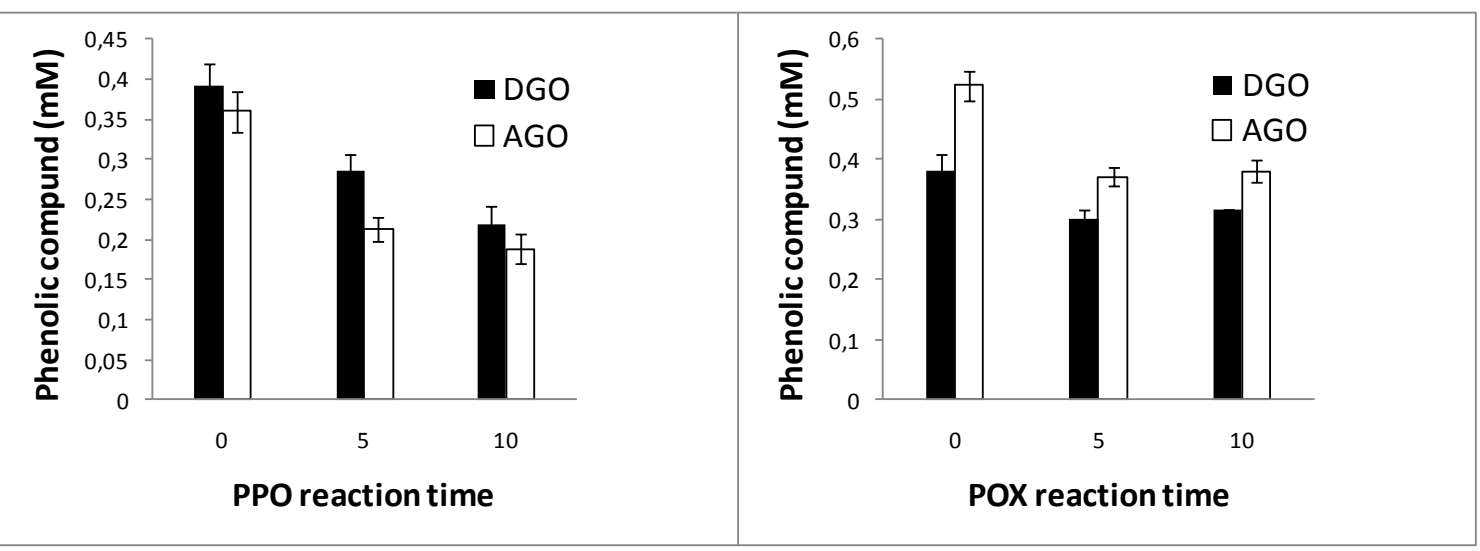

Figure 3.- Kinetic of ortho-diphenolic secoiridois, DGO and AGO, by olive POX and olive PPO. 